\title{
Sliding mode attitude control for flexible spacecraft
}

\author{
Wang Qi \\ Nanchang Hangkong University \\ Nanchang Jiangxi, China \\ wangqi439@126.com
}

\author{
Wei Qin \\ Nanchang Hangkong University \\ Nanchang Jiangxi, China \\ welkinwq@163.com
}

\begin{abstract}
Aiming at the attitude control problem of flexible spacecraft, an improved sliding mode controller is presented. First of all, the dynamics equation and the kinematics equation described by error quaternion and error angular velocity are studied, which avoid the non-single question of the end attitude. What's more, according to the mathematical model of flexible spacecraft attitude system, the sliding mode controller is designed by the Lyapunov approach. Finally, in order to reduce the chattering of variable structure control for flexible spacecraft attitude, the smoother "arc tan function" is used to replace the sign function to reduce the chattering. And optimization to reduce the maximum control torque required by the system, which can avoid the vibration of flexible appendages. Simulation results show that the proposed control scheme can effectively achieve the spacecraft attitude control. Besides, On account of this, we can find that it can eliminate the chattering of traditional sliding mode control and keep good robustness.
\end{abstract}

Keywords-flexible spacecraft; error quaternion; sliding mode control; Chattering reduction

\section{INTRODUCTION}

With the continuous development of space technology, the solar battery panels, mechanical arms, aerial and other large-scale flexible accessories are usually with the latest generation spacecraft. Among these types of spacecraft, while flying and controlling as the result of a strong coupling exist between the rigid body and flexible body which makes the control force on the spacecraft not only cause the change of the spacecraft attitude but also lead to a continuous chattering of the flexible structure. And these would affect the locate accuracy of spacecraft attitude on one hand, and critical the normal operating of interior instruments even lead the failure of the spacecraft on the other hand. Therefore, it is necessary to look for a controlling method which can both control the attitude and reduce the robustness caused by the chattering of flexible structure while designing the attitude control coefficient of the flexible spacecraft.

As one of the variable structure control method, sliding variable structure control is insensitive to the external disturbance which satisfied the match condition and the uncertainty of parameters, due to good robustness and other advantages, it gets a widespread application on the spacecraft attitude control ${ }^{[1-3]}$. However, the system chattering is an obvious weak point of the various structure control, if this chattering cannot be eliminated or weaken, then the chattering of the flexible accessory may endure for long time which greatly reduced the meaning of various structure control. Reference [4] proposed using saturation function to replace sign function in order to reduce the chattering can be an effective way, but to flexible spacecraft will still cause the continuous chattering of the flexible accessories. GuanPing etc ${ }^{[5]}$ use the fuzzy various structure control method, considering the uncertainty and external disturbance to solve the issue of flexible satellite attitude control, but these methods haven't completely eliminate all kinds of uncertainty among the system, besides, the control algorithm is complicated.

This paper has used the quaternion method to build the mathematic model of flexible spacecraft. Then design the attitude control coefficient of the sliding various structure according to the Lyapunov approach. We use the "arc tan function" to replace the original switch function, so that the high frequency chattering can be reduced. And through optimizing the control quantity to avoid the flexible accessories chattering of the spacecraft caused by oversized control torque when maneuvering. At the end of the paper, the proposed control algorithm has been conducted the mathematic simulation, and the result has proved the effectiveness of the algorithm.

\section{MATHEMATIC MODEL BUILDING OF FLEXIBLE SPACECRAFT}

The dynamic model and kinematic model of flexible spacecraft are as follow ${ }^{[6]}$ :

$$
\begin{gathered}
J \dot{w}+w^{\times} J w+\delta^{T} \ddot{\eta}=u+d \\
\ddot{\eta}+2 \xi \Lambda \dot{\eta}+\Lambda^{2} \eta+\delta \dot{w}=0 \\
\dot{q}=\frac{1}{2} F(q) w
\end{gathered}
$$

Among these parameters, $J$ denotes the rotary inertia of the spacecraft, $w$ denotes the angular velocity vector, $u$ denotes the control torque, $d$ denotes the perturbed torque, $\delta$ denotes the coupling coefficient matrix of the spacecraft and flexible accessories, $\eta$ denotes the chattering model coordinate of the flexible accessories, $\xi$ denotes the chattering damping ratio matrix of the flexible accessories, $\Lambda$ denotes the chattering frequency matrix of the flexible accessories, and $w^{\times}$denotes the skew symmetric matrix of $w, q=\left[\begin{array}{llll}q_{0} & q_{1} & q_{2} & q_{3}\end{array}\right]^{T}$ denotes the quaternion vector, and also $\|q\|=1$, the expression of $\mathrm{F}(\mathrm{q})$ is: 


$$
F(q)=\left[\begin{array}{c}
-q_{v}^{T} \\
q_{v}^{\times}+q_{0} I_{3}
\end{array}\right]
$$

Where $I_{3}$ denotes a $3 \times 3$ unit matrix, $q_{v}=\left[\begin{array}{lll}q_{1} & q_{2} & q_{3}\end{array}\right]^{T}$ $q_{v}^{\times}$denotes the skew symmetric matrix of $q_{v}$.

Assuming the expectation attitude quaternion is $q_{d}$ and define the operating attitude quaternion of spacecraft is $q$, then according to the synthesis method of quaternion ${ }^{[7]}$ :

$$
q=q_{d} \otimes q_{e} \Rightarrow q_{e}=q_{d}^{-1} \otimes q
$$

Where, $q_{e}$ denotes error quaternion, ${ }^{\otimes}$ denotes the quaternion multiplier, and then based on (5):

$$
q_{e}=\left[\begin{array}{c}
q_{e 0} \\
q_{e 1} \\
q_{e 2} \\
q_{e 3}
\end{array}\right]=\left[\begin{array}{cccc}
q_{d 0} & q_{d 1} & q_{d 2} & q_{d 3} \\
-q_{d 1} & q_{d 0} & q_{d 3} & -q_{d 2} \\
-q_{d 2} & -q_{d 3} & q_{d 0} & q_{d 1} \\
-q_{d 3} & q_{d 2} & -q_{d 1} & q_{d 0}
\end{array}\right]\left[\begin{array}{l}
q_{0} \\
q_{1} \\
q_{2} \\
q_{3}
\end{array}\right]
$$

When, $q=q_{d}, q_{e}=\left[\begin{array}{llll}1 & 0 & 0 & 0\end{array}\right]^{T}$.Therefore, the spacecraft attitude tracking problem can be described as the certain spacecraft initial attitude quaternion $q$ and expectation attitude quaternion $q_{d}$, and designing the control device to make error quaternion adjust to $q_{e}=\left[\begin{array}{llll}1 & 0 & 0 & 0\end{array}\right]^{T}$.

However, the general initial attitude and expectation attitude we proposed are usually based on Euler angle as an attitude description object. The conversion equations of Euler angle and quaternion are following ${ }^{[8]}$ :

$$
\begin{gathered}
q_{0}=\cos (\phi / 2) \cos (\theta / 2) \cos (\psi / 2)+\sin (\phi / 2) \sin (\theta / 2) \sin (\psi / 2) \\
q_{1}=\sin (\phi / 2) \cos (\theta / 2) \cos (\psi / 2)-\cos (\phi / 2) \sin (\theta / 2) \sin (\psi / 2) \\
q_{2}=\cos (\phi / 2) \sin (\theta / 2) \cos (\psi / 2)+\sin (\phi / 2) \cos (\theta / 2) \sin (\psi / 2) \\
q_{3}=\cos (\phi / 2) \cos (\theta / 2) \sin (\psi / 2)-\sin (\phi / 2) \sin (\theta / 2) \cos (\psi / 2) \\
\theta=\arcsin \left(-2\left(q_{1} q_{3}-q_{0} q_{2}\right)\right) \\
\phi=\arctan \left(\frac{2\left(q_{2} q_{3}+q_{0} q_{1}\right)}{q_{0}^{2}-q_{1}^{2}-q_{2}^{2}+q_{3}^{2}}\right) \\
\psi=\arctan \left(\frac{2\left(q_{1} q_{2}+q_{0} q_{3}\right)}{q_{0}^{2}+q_{1}^{2}-q_{2}^{2}-q_{3}^{2}}\right)
\end{gathered}
$$

Where, $\theta$ is the pitch angle, $\phi$ denotes the rolling angle, $\psi$ is the drift angle.

Assuming the operating attitude angular velocity is $w$, the expectation attitude angular velocity is $w_{d}$, then the error of attitude angular velocity defines:

$$
w_{e}=w-w_{r}
$$

Where, $w_{r}=A\left(q_{e}\right) w_{d}, A\left(q_{e}\right)$ denotes the transition matrix from orbit coordinate system to body coordinate system which based on the description of error quaternion. The expression is as follow:

$$
A\left(q_{e}\right)=\left(q_{0 e}^{2}-q_{v e}^{T} q_{v e}\right) I_{3}-2\left(q_{0 e} q_{v e}^{\times}-q_{v e} q_{v e}^{T}\right)
$$

Besides:

$$
\dot{w}_{r}=A\left(q_{e}\right) \dot{w}_{d}-w_{e}^{\times} A(q e) w_{d}
$$

Reference [3], in order to calculate conveniently, combine the external disturbance $d$ and rigid-flexible coupling $\delta^{T} \ddot{\eta}$ together as the total inference term $D, D=d-\delta^{T} \ddot{\eta}$, then the (1) can rewrite as :

$$
J \dot{w}+w^{\times} J w=u+D
$$

From the $(1) \sim(10)$, the flexible spacecraft attitude error equation is:

$$
J \dot{w}_{e}=-J \dot{w}_{r}-w^{\times} J w+u+D
$$

The error quaternion can express the dynamic model as follow:

$$
\dot{q}_{e}=\frac{1}{2} E\left(q_{e}\right) w_{e}
$$

Where: $E(q)=\left[\begin{array}{c}-q_{v e}^{T} \\ q_{v e}^{\times}+q_{0 e} I_{3}\end{array}\right], q_{v e}=\left[\begin{array}{lll}q_{e 1} & q_{e 2} & q_{e 3}\end{array}\right]^{T}$.

\section{DESIGN OF THE SLIDING MODE CONTROLLER}

First of all, define the switch surface that the sliding various structure control needed, and the switch surface general choose a variance error and its derivative, according to the attitude mathematic model of the spacecraft, the switch surface function can be defined as:

$$
S=w_{e}+k q_{v e}
$$

Among the formula:

$$
S=\left[\begin{array}{lll}
S_{1} & S_{2} & S_{3}
\end{array}\right]^{T}, k=\operatorname{diag}\left\{\begin{array}{lll}
k & k & k
\end{array}\right\}, k>0 .
$$

The existence of the sliding modal is the using premise of sliding various structure control, in order to ensure the existence of sliding modal, the following condition must be satisfied, which is:

$$
S \dot{S}<0
$$

Aiming to the non-linearity dynamic equation of flexible spacecraft attitude (13), use the equivalent control method ${ }^{[10]}$ to design the sliding modal control coefficient $u$ as:

$$
u=u_{s w}+u_{e q}
$$

Among the control of equivalent sliding various structure, the control coefficient usually composed by the equivalent control $u_{e q}$ and switch control $u_{s w}$, The equivalent control keep the system condition on the sliding surface and switch control force the system condition won't come out of the sliding surface.

In order to ensure the establishment of the sliding mode reaching the match condition, the design of switch control is as follow:

$$
u_{s w}=-K_{1} S-D_{1} F(S)
$$

Where,

$$
K_{1}=\operatorname{diag}\left\{k_{i}\right\}, D_{1}=\operatorname{diag}\left\{d_{i}\right\} k_{i}>0, d_{i}>0, \quad i=1,2,3 \text {. }
$$

These are the control parameters.

$$
F(S)=\left\{\operatorname{sgn}\left(S_{1}\right), \operatorname{sgn}\left(S_{2}\right), \operatorname{sgn}\left(S_{3}\right)\right\}^{T}
$$

Here the $\operatorname{sgn}\left(S_{i}\right)$ is symbol function.

The $u_{e q}$ denotes the equivalent controls, while $\dot{S}=0$, can get the following result:

$$
u_{e q}=J \dot{w}_{r}+w^{\times} J w-J k \dot{q}_{v e}
$$


When the parameter $D_{l}$ of the control device satisfied $\min \left\{D_{1}\right\}>\|D\|$, the designed control coefficient (17) can stabilize the system.

Prove the above. Define the condition of (16) to Lyapunov function, and select Lyapunov function which is ${ }^{[10]}$ :

$$
V=\frac{1}{2} S^{T} J S
$$

Derivate (21), can get the following result:

$$
\begin{aligned}
& \dot{V}=S^{T} J \dot{S}=S^{T} J\left(\dot{w}_{e}+k \dot{q}_{v e}\right) \\
& =S^{T}\left(-w^{\times} J w-J w_{r}+u+D+J k \dot{q}_{v e}\right)
\end{aligned}
$$

From the control theory we can know, when $\dot{V}<0$, the system is stable, use (17) substitute (22), can get:

$$
\dot{V}=S^{T} J \dot{S}=S^{T}\left[D-K_{1} S-D_{1} F(S)\right]<0
$$

So, $\dot{V}<0$ is been proved.

\section{OPTIMIZE THE SLIDING MODEL CONTROLLER}

Considering the flexible accessories of flexible spacecraft has some features like low damping, low frequency and multi-modal, so when design the controller should try to avoid stimulating the accessories chattering. From the chattering (2) of the flexible accessories, we can know, $\delta \dot{w}$ has a direct relation flexible accessories, which is angular acceleration. If $\dot{w}$ can be decreased and diffused, then the chattering of flexible accessories is able to be reduced effectively.

However, $\dot{w}$ is directly related to the control torque, therefore, conducting the optimization of control coefficient need to considerate. Through analyzing the control coefficient(17) which designed above can find out that, the symbol function in switch control is the important reason for generating the chattering under the control of sliding various structure, due to the very cliffy change of the symbol function, it's very easy to cause the flexible accessories chattering. Besides, at the initial time when the flexible spacecraft do the attitude maneuvering, the attitude error is large, so that a comparatively large control torque is needed by the control coefficient. These will not only increase the output torque of the spacecraft itself actuator requirement, but also cause the flexible accessories chattering.

To the questions mentioned above, we have improved the control (17) through using saturation function or the continuous function with relay characteristic ${ }^{[11]}$ to replace the symbol function which is in the ideal sliding modal. Although by replacing symbol function with saturation function can reduce the chattering, but the saturation function still very cliffy comparatively to cause the chattering of the flexible accessories. Here lead in the arc tan function which comparatively smoother to solve the mentioned problems.

Command $F_{1}(S)=\left[f\left(S_{1}\right), f\left(S_{2}\right), f\left(S_{3}\right)\right]^{T}$, where $f\left(S_{i}\right)$ is the improved arc tan function.

$$
f\left(S_{i}\right)=\left\{\begin{array}{cc}
1, & S_{i}>1 \\
\arctan \left(x S_{i}\right), & -1 \leq S_{i} \leq 1, x=\tan (1)=1.5574,(i=1,2,3) \\
-1, & S_{i}<-1
\end{array}\right.
$$

Besides, we need add an item $a$ which can generate the delay effect in the switch control items,

where $a=\operatorname{diag}\left\{a_{i}\right\}, i=1,2,3$.

$$
a_{i}=\left(1+\lambda-e^{-\beta t}\right)
$$

$a$ is approaching to 1 as the form of exponential function, the delay effect stops when its approached 1 and the duration of delay effect is decided by $\beta$, and $\lambda$ is a very small figure mainly to avoid the situation of generating 0 at the initial time. After adding the delay factor, the comparatively large overshoot caused during the initial time is been avoid, and the maximum control torque is been reduced, meanwhile, the caused chattering of spacecraft flexible accessories is also been reduced. After going through the initial stage, with the time increasing, the figure is approaching to 1 , and the delay effect is been weakened and eliminated, also the control coefficient is back to the original sliding various structure control, the flexible spacecraft continue conducting attitude maneuver at a fast speed, the control coefficient output is more smooth, the changing is more stable, and the chattering of flexible accessories is more less.

So the improved control coefficient is:

$$
\begin{gathered}
u=u_{s w}+u_{e q}=-a K_{1} S-D_{1} F_{1}(S)+u_{e q} \\
\text { V. SimULATION ANALYZING }
\end{gathered}
$$

Use MATLAB to stimulate the attitude control of flexible spacecraft. Initial conditions are as follow: Rotary inertia matrix of spacecraft is:

$$
J=\left[\begin{array}{ccc}
5114.65 & 21.56 & -16.87 \\
21.56 & 3789.84 & 1494.78 \\
-16.87 & 1494.78 & 6688.91
\end{array}\right]\left(\mathrm{kg} \cdot \mathrm{m}^{2}\right)
$$

Coupled matrix of flexible accessories and the main body of spacecraft is :

$$
\delta=\left[\begin{array}{ccc}
0.3537 & -0.0131 & 27.5129 \\
17.3123 & -21.5032 & 0.0726 \\
-19.6524 & -25.2704 & 0.5721
\end{array}\right]\left(\mathrm{kg}^{1 / 2} \cdot \mathrm{m}\right)
$$

Chattering frequency matrix of flexible accessories is:

$\Lambda=\operatorname{diag}\left\{\begin{array}{lll}1.03246 & 1.22528 & 1.87637\end{array}\right\}(\mathrm{rad} / \mathrm{s})$

Chattering damp matrix of flexible accessories is:

$$
\xi=\operatorname{diag}\left\{\begin{array}{lll}
0.001 & 0.001 & 0.001
\end{array}\right\}
$$

Perturbed torque is :

$$
D=\left[\begin{array}{c}
6.8 \cos t \\
1.4 \sin t-2.8 \cos t \\
-1.4 \sin t
\end{array}\right] \times 10^{-3}(N \cdot m)
$$

Control device parameter choice is: 


$$
\begin{aligned}
& K_{1}=\operatorname{diag}\left\{\begin{array}{lll}
1200 & 1200 & 1200
\end{array}\right\} \\
& D_{1}=\operatorname{diag}\left\{\begin{array}{lll}
0.85 & 0.85 & 0.85
\end{array}\right\}
\end{aligned}
$$

The attitude Euler angle at initial time is $\left[\begin{array}{lll}3^{\circ} & -5^{\circ} & 7^{\circ}\end{array}\right]^{T}$, the expectation attitude Euler angle is $\left[\begin{array}{lll}0^{\circ} & 0^{\circ} & 0^{\circ}\end{array}\right]^{T}$, both the initial and expectation attitude angular velocity is $\left[\begin{array}{lll}0 & 0 & 0\end{array}\right]^{T}(\% / s) . k$ is $1, \beta$ is $0.1, \lambda$ choose 0.001 . The simulation result is as the following chart:

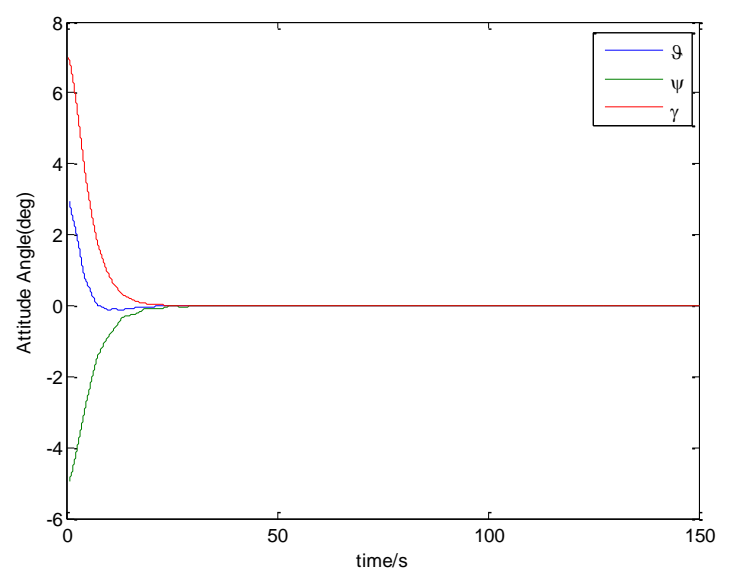

Figure 1. Unoptimizable attitude angular respond curve

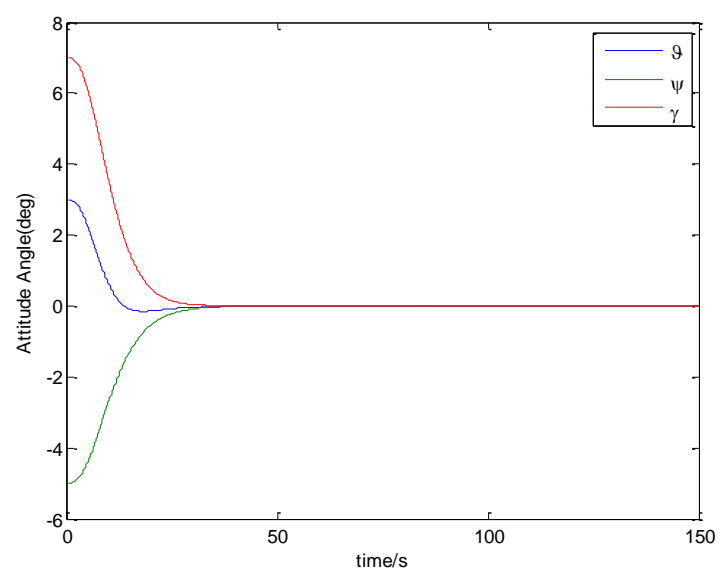

Figure 2. Optimized attitude angular respond curve

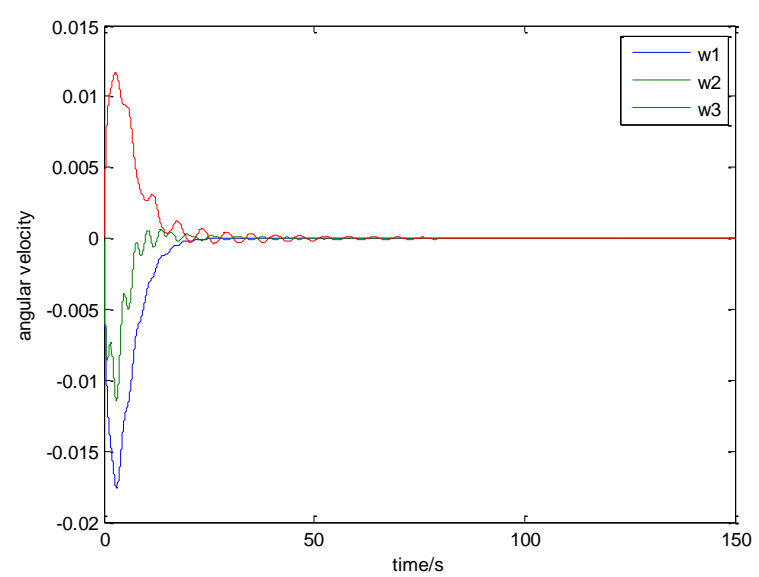

Figure 3. Unoptimizable attitude angular velocity respond curve

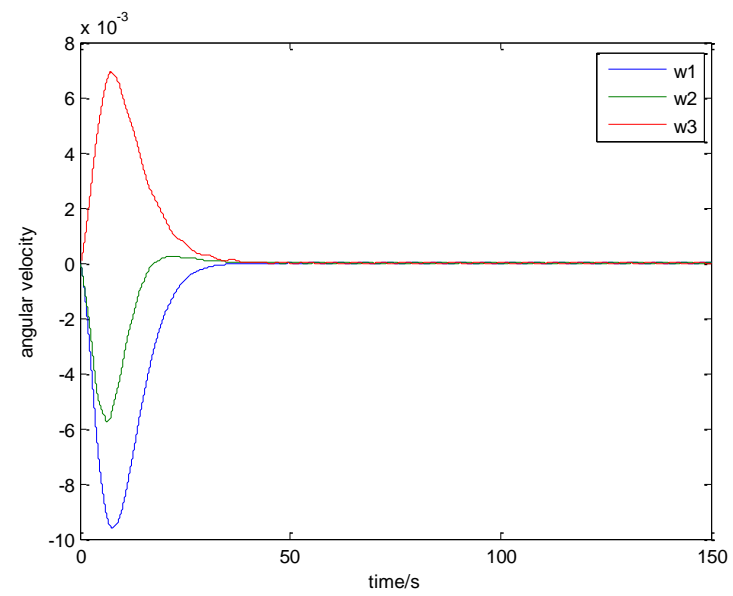

Figure 4. Optimized attitude angular velocity respond curve

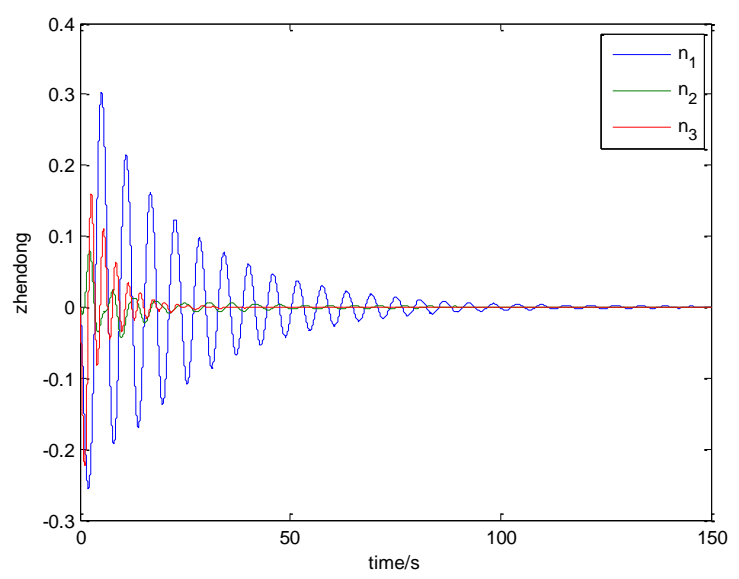

Figure 5. Unoptimizable chattering modal of flexible accessories 


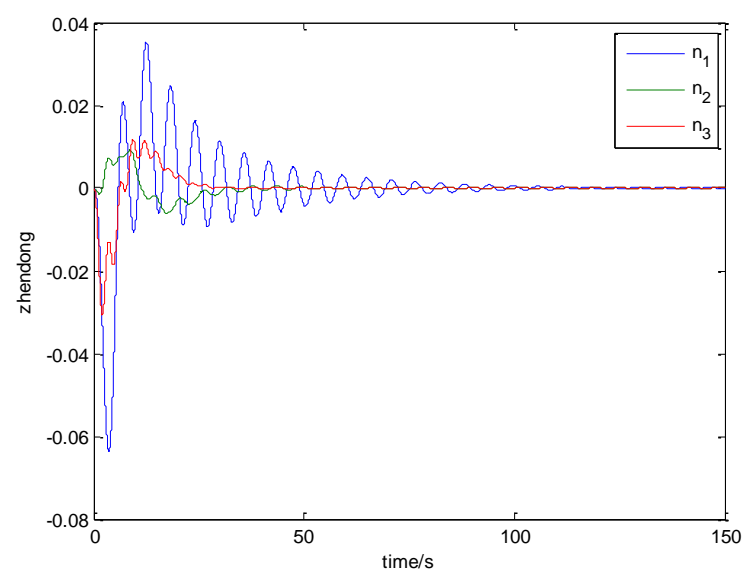

Figure 6. Optimized chattering modal of flexible accessories
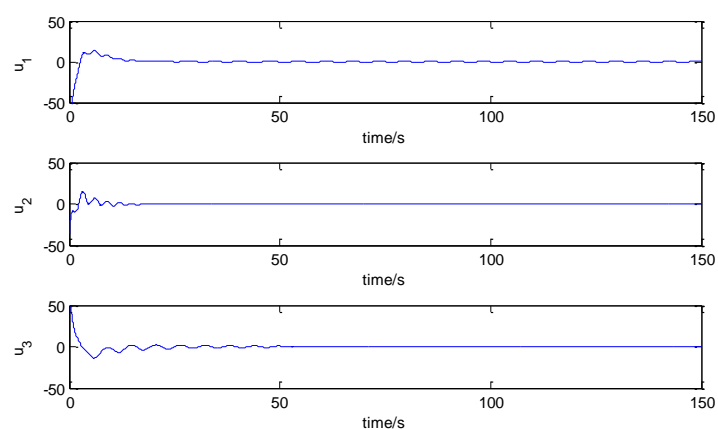

Figure 7. Unoptimizable control torque
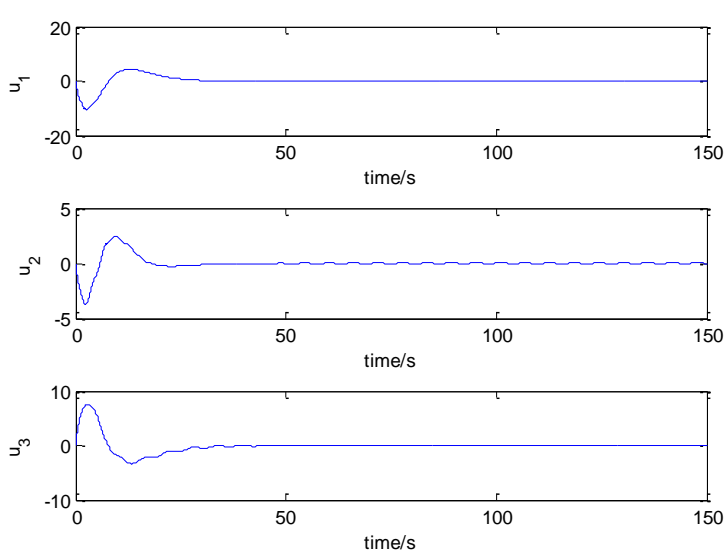

Figure 8. Optimized control torque respond curve

From Fig .1 and Fig .2 we can know, both of the algorithm can guarantee the attitude maneuver assignment of spacecraft, and optimized control coefficient of sliding various structure has a little increased on the maneuver time, but the maneuver route is more stable; Compare with Fig .3 and Fig .4, we can know that optimized control coefficient of sliding various structure can decrease the maximum attitude angular velocity, and also more stable; Compare with Fig .5 and Fig .6, the optimized sliding control coefficient has restrained the chattering of flexible accessories very effectively, and make the chattering amplitude deceased almost one order of magnitude compare with the unoptimizable; Compare with Fig .7 and Fig .8, we can see that to accomplish the same attitude maneuver movement, the required maximum torque of optimized algorithm has been decreased couple times compare with the unoptimizable algorithm.

\section{CONCLUSION}

The stimulation result of the flexible spacecraft attitude maneuver control shows, the effectivity of the optimization method, a better attitude tracking of flexible spacecraft has been fulfilled, the directed accuracy and stability of the maneuvered flexible spacecraft has also been greatly improved. Applying the method of this paper can reduce the chattering, improve the stability of attitude maneuvering, restrain the chattering of flexible accessories, and reduce the requirement of maximum output torque on executive body greatly, and the control ability surpass the traditional sliding various structure control. The designing method of control device in this paper has actual meaning to the attitude control of flexible spacecraft.

\section{REFERENCES}

[1] Hu Q, Ma G. Control of three-axis stabilized flexible spacecraft using variable structure strategies subject to input nonlinearities[J]. Journal of Vibration and Control, 2006, 12(6): 659-681.

[2] Hu Q. Sliding mode maneuvering control and active vibration damping of three-axis stabilized flexible spacecraft with actuator dynamics[J]. Nonlinear Dynamics, 2008, 52(3): 227-248.

[3] Lo S C, Chen Y P. Smooth sliding-mode control for spacecraft attitude tracking maneuvers[J]. Journal of Guidance, Control, and Dynamics, 1995, 18(6): 1345-1349.

[4] Lv J T,Li C J,Ma G F.A sliding mode PID controller design for satellite attitude regulation[J].Journal of Harbin Institute of Technology,2008,40(7):1009-1012.

[5] Guan P, Liu X H, Liu X J.Variable structure attitude control of flexible spacecraft $[\mathrm{J}]$. Control Theory \& Applications, 2007,24(3):480-484

[6] Tu S C. The satellite attitude dynamics and control [M] China Astronautic Publishing House .1998.(in Chinese).

[7] Zhang R H, Jia H G, Chen T. Attitude solution for strapdown intertial navigation system based on quaternion algorithm [J].Optics and Precision Engineering,2008,10(16),1963-1970

[8] Huang R W. Satellite orbit and attitude dynamics and control [M] Beihang University Press. 1998.(in Chinese)

[9] Hu Q. Variable structure maneuvering control with time-varying sliding surface and active vibration damping of flexible spacecraft with input saturation[J]. Acta Astronautica, 2009, 64(11): 10851108.

[10] Liu J K, Sun F C. Research and development on theroy and algorithms of sliding mode control [J]. Control Theory \& Applications ,2009, 24(3), 407-418

[11] Lo S C, Chen Y P. Smooth sliding-mode control for spacecraft attitude tracking maneuvers[J]. Journal of Guidance, Control, and Dynamics, 1995, 18(6): 1345-1349.

[12] I.J.Cheon,Aquaternion approach to variable structure control of spacecraft attitude tracking, Annual Spring Meeting of The Korean Society for Aeronautical and SpaceScience, Cheongju,Korea,2000,211-214. 